\title{
Docencia sobre las fuentes comerciales de información de medicamentos y opinión de los estudiantes de medicina
}

\author{
A. Vallano, C. Aguilera, A. Figueras
}

Objetivo. Conocer la opinión de los estudiantes sobre un seminario que enseña la interacción de los médicos con las fuentes comerciales de información de los medicamentos. Sujetos y métodos. Se realizó una encuesta a los estudiantes de tercero de medicina sobre un seminario que trataba de las fuentes comerciales de información de medicamentos. El seminario incluyó una videograbación de un representante comercial de una empresa farmacéutica que presentaba un nuevo medicamento a un médico, y ejemplos de información sobre medicamentos incluida en el catálogo comercial Vademécum Internacional y en los anuncios publicitarios. Se preguntó a los estudiantes el grado de interés y de satisfacción con el seminario y qué nivel de utilidad creían que podían tener las actividades desarrolladas en el seminario en su futuro profesional. En la valoración de la opinión se utilizó una escala de Likert (de 0 a 10 puntos). Resultados. De 107 estudiantes que asistieron a los seminarios, 101 (94\%) participaron en la encuesta. La edad media de los estudiantes fue de 21 años (desviación estándar -DE-: 2,2 años) y 75 fueron mujeres (74\%). La puntuación media del grado de interés fue de 7,4 (DE: 1,5), del grado de satisfacción de 7,5 (DE: 1,6) y del grado de potencial utilidad de 7,6 (1,6). Conclusión. Los estudiantes de medicina están interesados en las actividades docentes sobre las fuentes de información comercial, y consideran que pueden ser potencialmente útiles para su futuro profesional. Es necesario formar y preparar a los estudiantes de medicina para la interacción con las fuentes comerciales de medicamentos.

Palabras clave. Actitud del personal de salud. Educación. Estudiantes. Industria farmacéutica. Médicos. Universitario.

\section{Teaching on the commercial drug}

information and medical students opinion

Aim. To know the opinion of the students about the teaching activities carried out in a seminar about the interaction of phy- sicians with the drug information sources from pharmaceutical companies. Subjects and methods. A survey was carried out to know the opinions from third year medical students after a seminar about drug information sources. The seminar included a video showing an encounter between a physician and a pharmaceutical company representative and further critical discussion of this interaction as well as other sources from pharmaceutical companies (drug commercial catalogue and advertisements). The information was gathered by means of a questionnaire that included variables related to the students' opinions about the interest, the satisfaction and utility on knowledge and skills acquired in seminar by means of a ten points Likert scale. Results. A total of 107 students attended the seminars and 101 (94\%) participated in the survey. The mean age of students was 21 (standard desviation -SD-: 2.2) years and 75 were women (74\%). The mean score of interest in the seminar was 7.4 (SD: 1.5), the mean score of satisfaction was 7.5 (SD: 1.6) and the mean score of utility was 7.6 (SD: 1.6). Conclusion. Medical students are interested in this teaching activity on commercial drug information, they are satisfied with the activity, and they consider it to be useful. It is necessary to educate the students of medicine for the interaction with the marketing activities from pharmaceutical companies.

Key words. Attitude of health personal. Drug industry. Education. Medical. Students. Undergraduate.

\section{Introducción}

Las fuentes de información sobre los medicamentos que tienen los médicos pueden determinar sus actitudes y sus pautas de prescripción. Los estudiantes de medicina tienen que conocer las diferentes fuentes de información de medicamentos disponibles, tanto profesionales como comerciales, y las características de la información que proporcionan. Hay datos que indican que
Fundació Institut Català de Farmacologia. Departament de Farmacologia, Terapèutica i Toxicologia. Unitat docent. Hospital Universitari Vall d'Hebron. Universitat Autònoma de Barcelona. Barcelona, España.

\section{Correspondencia}

Dr. Antonio Vallano Ferraz. Fundació Institut Català de Farmacologia.

Servicio de Farmacología Clínica. Hospital Universitari Vall d'Hebron. Pg. Vall d'Hebron, 119-129.

E-08035 Barcelona.

Fax

+34934894109

E-mail

tv@icf.uab.es

Aceptación definitiva 08.05.2008. 
las fuentes comerciales de información sobre los medicamentos, elaboradas por las empresas farmacéuticas, tienen una gran influencia en el proceso de prescripción [1-7]. La educación de los estudiantes de medicina tiene que incluir la formación sobre las fuentes comerciales de los medicamentos y preparar a los futuros médicos para gestionarlas adecuadamente. Hay pocos estudios publicados que describan iniciativas educativas de formación de los estudiantes de medicina sobre las fuentes comerciales de información de los medicamentos [8] y no hemos identificado ningún estudio que evalúe la opinión de los estudiantes sobre el desarrollo de estas actividades docentes. El objetivo del estudio fue evaluar la opinión que tienen los estudiantes sobre las actividades educativas relacionadas con las fuentes comerciales de información de medicamentos.

\section{Sujetos y métodos}

\section{Estudio de diseño}

Se realizó una encuesta voluntaria y anónima a los estudiantes de tercer año de la licenciatura de medicina para conocer su opinión sobre un seminario que trataba las fuentes comerciales de información de medicamentos y que se hacía dentro del programa docente de la asignatura de farmacología. La encuesta se hizo a los estudiantes que participaron en los seminarios en dos cursos consecutivos (enero de 2006 y enero de 2007).

\section{Contenido del seminario}

Los seminarios tenían como objetivo docente formar a los estudiantes para que conocieran las fuentes comerciales de información de medicamentos diferentes y sus características principales. Los seminarios duraban tres horas aproximadamente $y$ se presentaban tres tipos de fuentes comerciales de medicamentos: los visitadores médicos de las empresas farmacéuticas, los catálogos comerciales de medicamentos y los anuncios de medicamentos. Primero, el seminario incluía una videograbación de 15 minutos de duración que mostraba una simulación de una reunión entre un visitador médico y un médico. El vídeo trataba de un visitador médico de una empresa farmacéutica ficticia que presentaba un 'nuevo' antiinflamatorio no esteroideo, que también era ficticio, y que, además, durante el encuentro ofrecía diversos regalos al médico. Después se realizaba un debate de grupo en el que se analizaban las técnicas de marketing directo y el contenido de los mensajes de promoción comercial del nuevo medicamento. Se comentaba la información que daba el visitador médico sobre el nuevo medicamento, pero también se comentaba la información que no daba y se reflexionaba sobre los regalos que ofrecía éste al médico. Posteriormente, también se presentaban y se comentaban las características y la calidad de información de los medicamentos contenida en el catálogo comercial Vademécum Internacional y en los anuncios publicitarios de medicamentos con ejemplos concretos.

\section{Variables}

La entrevista se realizó con un cuestionario estructurado que incluía variables demográficas de los estudiantes y sus opiniones sobre el grado de interés y de satisfacción con el seminario y el grado de potencial utilidad del conocimiento adquirido para la práctica clínica. Se solicitó también el grado de interés en fuentes comerciales específicas como la visita médica, el catálogo comercial Vademécum Internacional (www.vademecum.es) y los anuncios publicitarios de medicamentos. La encuesta también pedía la opinión sobre el nivel de satisfacción y el nivel de utilidad percibida de la videograbación. Además, la encuesta pedía el grado de satisfacción con el análisis crítico de la información contenida en el catálogo comercial y en los anuncios publicitarios. En la valoración de las opiniones se utilizó una escala Likert (de 0 a 10 puntos). También se preguntó la opinión sobre el tiempo dedicado a estos temas en el seminario con una escala de tres categorías (insuficiente, suficiente y excesivo).

\section{Aspectos éticos}

La participación de los estudiantes en la encuesta era voluntaria y anónima, y se destacó de manera explícita que su implicación en la misma no tenía ninguna relación con el proceso de evaluación de la asignatura.

\section{Análisis estadístico}

La estadística fue descriptiva (frecuencia, media, desviación estándar, mediana y márgenes). Las di- 
Tabla I. Grado de interés, satisfacción y utilidad de los seminarios según la opinión de los estudiantes.

\begin{tabular}{cccc}
\hline Puntuación & Interés, $n(\%)$ & Satisfacción, $n(\%)$ & Utilidad, $n$ (\%) \\
\hline 0 & - & - & $1(1,0)$ \\
\hline 1 & - & $1(1,0)$ & - \\
\hline 2 & $1(1,0)$ & $2(2,0)$ & - \\
\hline 3 & - & - & $7(6,9)$ \\
\hline 4 & $2(2,0)$ & $6(5,9)$ & $16(15,8)$ \\
\hline 5 & $5(5,0)$ & $12(11,9)$ & $22(21,8)$ \\
\hline 7 & $15(14,9)$ & $25(24,8)$ & $25(24,8)$ \\
\hline 8 & $32(31,7)$ & $34(33,7)$ & $15(14,9)$ \\
\hline 10 & $25(24,8)$ & $12(11,9)$ & $15(14,9)$ \\
\hline
\end{tabular}

ferencias entre dos o más variables continuas se compararon por medio del test ANOVA. El nivel de significación estadística fue de $p<0,05$. El análisis estadístico se realizó con el programa estadístico SPSS.

\section{Resultados}

Un total de 256 estudiantes (125 estudiantes en el año 2006 y 131 estudiantes en el año 2007) se matricularon en la asignatura de farmacología durante el tercer año de licenciatura. De los 107 estudiantes que asistieron a los seminarios (un $41,8 \%$ de todos los estudiantes matriculados), 101 $(94,4 \%)$ participaron en la encuesta. Sesenta y dos y 39 estudiantes participaron en los años 2006 y 2007 , respectivamente. La edad media de los estudiantes fue de 21 (desviación estándar-DE-: 2,2) años y 75 (74\%) fueron mujeres.

La puntuación media del grado de interés del seminario fue de 7,4 (DE: 1,5 ), del grado de satisfacción de 7,5 (DE: 1,6) y del grado potencial de utilidad de 7,6 (DE: 1,6) (Tabla I). No había ninguna diferencia en el grado de interés, en el de satisfacción y en el de utilidad entre los estudiantes de los años 2006 y 2007, y tampoco entre los dos sexos. Respecto a la duración del seminario, 91 (90\%) estudiantes la consideraron suficiente, 7 $(6,9 \%)$ excesiva y $3(3 \%)$ insuficiente. Los estudiantes que valoraron como insuficiente o suficiente la duración del seminario estuvieron más interesados -puntuación media 7,5 (DE: 1,5)- que los que la consideraron excesiva -puntuación media 6,1 (DE: 1,2$) ; p=0,02)$-. Los estudiantes que consideraron insuficiente o suficiente la duración del seminario también pensaban que el seminario era más útil -puntuación media 7,7 (DE: 1,6)- que aquellos que la consideraban excesiva -puntuación media 6,3 (DE: 0,7); $p=0,02-$.

La puntuación media del grado de interés de la videograbación sobre la representación simulada del encuentro entre un visitador médico y un médico fue de 6,4 (DE: 2,0) -mediana 7, mínimo 0 y máximo $10-$, del grado de interés del análisis del catálogo comercial de medicamentos de 7,6 (DE: 1,7) -mediana 8, mínimo 1 y máxi- 
Tabla II. Grado de interés de las diferentes fuentes comerciales de medicamentos según la opinión de los estudiantes.

\begin{tabular}{cccc}
\hline Puntuación & $\begin{array}{c}\text { Visitador médico } \\
n(\%)\end{array}$ & $\begin{array}{c}\text { Vademécum internacional } \\
n(\%)\end{array}$ & $\begin{array}{c}\text { Anuncios publicitarios } \\
n(\%)\end{array}$ \\
\hline 0 & $1(1,0)$ & - & $12(11,9)$ \\
\hline 1 & $1(1,0)$ & - & $3(3,0)$ \\
\hline 2 & $3(3,0)$ & $2(2,0)$ & $10(9,9)$ \\
\hline 3 & $5(5,0)$ & $2(2,0)$ & $8(7,9)$ \\
\hline 4 & $5(5,0)$ & $2(2,0)$ & $27(26,7)$ \\
\hline 5 & $12(11,9)$ & $17(16,8)$ & $11(10,9)$ \\
\hline 6 & $20(19,8)$ & $22(21,8)$ & $18(17,8)$ \\
\hline 7 & $21(20,8)$ & $25(24,8)$ & $2(2,0)$ \\
\hline 8 & $21(20,8)$ & $13(12,9)$ & $5(5,0)$ \\
\hline 70 & $5(6,9)$ & $17,0)$ & $2(2,0)$ \\
\hline
\end{tabular}

mo 10-, y del grado de interés de los anuncios publicitarios de medicamentos de 4,7 (DE: 2,5) -mediana 5, mínimo 0 y máximo 10- (Tabla II). La puntuación media del grado de satisfacción de los estudiantes con la videograbación fue de 7,8 (DE: 1,6) -mediana 8, mínimo 0 y máximo $10-$ y el grado potencial de utilidad fue de 7,9 (DE: 1,7) -mediana 8, mínimo 1 y máximo 10- (Tabla III). Los estudiantes del año 2007 estuvieron más satisfechos con la cinta de vídeo -8,5 (DE: 1,5)- que los del año $2006-7,4$ (DE: 1,7); $p=0,003-$. Los estudiantes de 2007 también consideraban más útil $-8,5$ (DE: 1,6)- la simulación representada en la videograbación que los estudiantes del año 2006 $-7,6$ (DE: 0,7); $p=0,01-$. La puntuación media del grado de satisfacción con el análisis crítico de la información del catálogo comercial de medicamentos fue de 7,4 (DE: 1,5) -mediana 8, mínimo 1 y máximo 10-, y con el análisis crítico de los anuncios publicitarios de $7,1(1,9)$-mediana 7, mínimo 0 y máximo 10- (Tabla IV). Los estu- diantes del año 2007 estuvieron más satisfechos con el análisis crítico de anuncios -7,8 (DE: 1,3)que los del año $2006-6,8$ (DE: 2,1); $p=0,006$.

\section{Discusión}

Los resultados principales de nuestro estudio indican que los estudiantes universitarios están interesados en las actividades docentes que tratan sobre las fuentes comerciales de información de medicamentos, así como el conocimiento de las técnicas de marketing utilizadas por las empresas farmacéuticas para promover los medicamentos. Además, los estudiantes están satisfechos con estas actividades y las consideran potencialmente útiles para su futuro profesional. En España y en otros países desarrollados, gran parte de la información de medicamentos utilizada por los médicos viene de las actividades promocionales de la industria 
Tabla III. Grado de satisfacción y de potencial utilidad de la videograbación que simulaba un encuentro entre el visitador médico de una empresa farmacéutica y un médico, según la opinión de los estudiantes.

\begin{tabular}{ccc}
\hline Puntuación & $\begin{array}{c}\text { Satisfacción } \\
n(\%)\end{array}$ & $\begin{array}{c}\text { Utilidad } \\
n(\%)\end{array}$ \\
\hline 0 & $1(1,0)$ & - \\
\hline 1 & - & - \\
\hline 2 & - & - \\
\hline 3 & - & $2(1,0)$ \\
\hline 4 & $1(1,0)$ & $5(5,0)$ \\
\hline 5 & $4(4,0)$ & $12(11,9)$ \\
\hline 6 & $11(10,9)$ & $16(15,8)$ \\
\hline 7 & $26(25,7)$ & $26(25,8)$ \\
\hline 8 & $27(26,7)$ & $15(14,9)$ \\
\hline 9 & $7(6,9)$ & $24(23,8)$ \\
\hline 10 & $24(23,8)$ & \\
\hline
\end{tabular}

farmacéutica, pero hay muy poca información de las actividades docentes desarrolladas para tratar el tema de las fuentes comerciales de información de medicamentos [9]. Por eso, en la asignatura de farmacología incluimos un seminario sobre la influencia de las fuentes de información de las empresas farmacéuticas porque deseábamos preparar a los estudiantes para esta faceta de su vida profesional. Los seminarios estaban diseñados para generar un pensamiento crítico entre los estudiantes de medicina en cuanto a la influencia de los visitadores médicos de las empresas farmacéuticas y de otras fuentes comerciales sobre los médicos. Sin embargo, no sabíamos si los estudiantes médicos estarían interesados en este tema, satisfechos con esta actividad docente y considerarían esta actividad de enseñanza útil para su futuro profesional.

Pocos estudios han evaluado las opiniones de los estudiantes de medicina con respecto a la in-
Tabla IV. Grado de satisfacción con la revisión de la información del catálogo comercial de medicamentos y de los anuncios publicitarios de medicamentos, según la opinión de los estudiantes.

\begin{tabular}{ccc}
\hline Puntuación & $\begin{array}{c}\text { Vademécum } \\
\text { Internacional } \\
n(\%)\end{array}$ & $\begin{array}{c}\text { Anuncios } \\
\text { publicitarios } \\
n(\%)\end{array}$ \\
\hline 0 & - & $2(2,0)$ \\
\hline 1 & $1(1,0)$ & - \\
\hline 2 & - & - \\
\hline 3 & - & $1(1,0)$ \\
\hline 4 & $7(6,9)$ & $5(5,0)$ \\
\hline 5 & $16(15,8)$ & $17(16,8)$ \\
\hline 6 & $26(25,7)$ & $25(24,8)$ \\
\hline 7 & $31(30,7)$ & $27(26,7)$ \\
\hline 8 & $12(11,9)$ & $10(9,9)$ \\
\hline 10 & $8(7,9)$ & $10(9,9)$ \\
\hline
\end{tabular}

teracción con las empresas farmacéuticas $[10,11]$. Los resultados de un estudio que analizaba la opinión de estudiantes de medicina de la escuela de Harvard sugerían que la educación médica actual tiene un impacto limitado en la formación de los estudiantes sobre su futura interacción con las industrias farmacéuticas [11]. Es interesante destacar que muchos estudiantes (61\%) referían que no se consideraban formados adecuadamente sobre la interacción que debían tener como profesionales médicos con la industria farmacéutica. Además, algunas experiencias sugieren que los estudiantes de medicina corren un elevado riesgo de ser fácilmente influenciados por las campañas de marketing de las empresas farmacéuticas, pero la mayoría de éstos no ha recibido nunca formación sobre las técnicas de marketing farmacéutico [10]. Los resultados de una encuesta realizada a 1.143 estudiantes de tercer año de medicina de Estados 
Unidos mostraron que éstos recibían regalos de las empresas farmacéuticas o eran invitados por estas empresas a diferentes acontecimientos de promoción comercial, y además la mayoría de los estudiantes creían que tenían derecho a recibir estos regalos [12]. La Asociación Americana de Estudiantes de Medicina (AMSA) ha lanzado su campaña PharmFree a escala nacional para educar a los estudiantes de medicina sobre la influencia de la industria farmacéutica en el período de formación académica [13]. En otra encuesta realizada a los estudiantes de medicina finlandeses, la mayoría consideraba a la industria farmacéutica como una de sus fuentes de información de medicamentos más importantes [14]. La importancia de la promoción farmacéutica como fuente de información de los medicamentos y la intensidad de marketing farmacéutico aumentaba progresivamente durante el período de la licenciatura. La mayoría de los estudiantes creían que estas actividades afectarían a su conducta y hábitos de prescripción, y la conciencia de esta influencia aumentaba progresivamente durante la licenciatura [14]. Hay pocos estudios publicados sobre las iniciativas que describen intervenciones o medidas para educar a los estudiantes de medicina o de farmacia sobre la influencia de las actividades de promoción comercial de los medicamentos [8]. Sin embargo, en una encuesta reciente hecha por la Organización Mundial de la Salud (OMS) de un total de 1.041 facultades de medicina y de farmacia, 228 facultades ( 137 de medicina y 91 de farmacia) respondieron que estaban desarrollando actividades docentes sobre la promoción comercial de los medicamentos a sus estudiantes [15]. La encuesta constataba que muchos profesores de medicina y de farmacia reconocían la necesidad de formar sobre la promoción comercial de los medicamentos y habían incorporado este tema en sus programas, a menudo integrados en los cursos sobre terapéutica, farmacología o ética profesional, pero sólo una o dos horas lectivas [15]. La mayoría de los encuestados informaba que los materiales del curso frecuentemente eran presentaciones en PowerPoint o equivalentes [15]. En nuestro seminario utilizábamos una videograbación sobre un caso simulado de un encuentro entre un visitador médico de una empresa farmacéutica y un médico. Otros estudios ya han descrito el uso de vídeos educativos para estimular el debate de los temas de docencia entre los estudiantes $[16,17]$. Las películas se han utilizado como una herra- mienta educativa para aumentar la motivación de los estudiantes de medicina porque permiten entender mejor los conceptos de la farmacología clínica [18]. Los juegos de rol sobre la promoción comercial de los medicamentos que hacen los visitadores médicos también se han desarrollado para generar un pensamiento crítico de los estudiantes sobre estas actividades $[19,20]$. Además, las sesiones educativas sobre la evaluación crítica de anuncios publicitarios de medicamentos, tal como hicimos, también se han evaluado [21].

La educación de los estudiantes sobre la promoción comercial de los medicamentos podría cambiar sus actitudes y mejorar sus habilidades para manejarlas [7]. Las iniciativas docentes más sencillas como una clase de una hora y una discusión posterior o un taller específico podrían tener algunos efectos adecuados sobre estas actitudes $[20,22]$. No obstante, estas sesiones educativas de manera aislada pueden no cambiar significativamente las percepciones de los estudiantes sobre la influencia de la industria farmacéutica si no existe una política educativa institucional más amplia y consistente [23]. Así, los obstáculos más frecuentes al éxito de estas actividades docentes mencionadas son la falta de integración en el currículum, la asignación de una duración insuficiente a causa de la oposición de los otros miembros de las facultades y la asignación de una baja prioridad institucional a este tema [15]. Aunque muchos profesores tienen como objetivo docente de estas actividades la mejora de las conductas de prescripción, no hay estudios que hayan evaluado si la adquisición de estas habilidades en la evaluación crítica de la promoción comercial de los medicamentos están asociadas con una mejor prescripción $[7,15]$. Es necesaria la investigación que evalúe el efecto del conocimiento y las habilidades adquiridas en la evaluación crítica de las fuentes comerciales de promoción de los medicamentos sobre la calidad de la prescripción de éstos.

Últimamente cuatro asociaciones médicas (American Medical Student Association, Healthy Skepticism Inc, No Free Lunch y PharmAware) han recomendado cuatro objetivos para la educación sobre la promoción farmacéutica y de productos sanitarios: 1) educación explícita sobre la toma de decisiones y la evaluación de evidencia; 2) ayudar a entender que no hay ningún dato que indique que la promoción comercial de los medicamentos aporta más beneficios que perjuicios; 3 ) ayudar a entender la responsabilidad de los 
profesionales sanitarios para evitar la promoción comercial de los medicamentos y productos sanitarios, y 4) educación explícita sobre las fuentes de información fiables [24]. Dado que hay pruebas sobre la influencia de la promoción comercial de los medicamentos que hacen las compañías farmacéuticas en la práctica de los médicos y los patrones de prescripción, los currículums académicos de medicina tendrían que incluir una docencia formal que prepare a los estudiantes para evaluar críticamente estos contactos. La docencia de pregrado y la formación permanente de posgrado en el campo de la farmacología clínica son esenciales para evaluar la relación beneficio-riesgo de los medicamentos y coste-beneficio para promover una terapia más óptima y que esté más alejada de los intereses comerciales de las compañías farmacéuticas. Por desgracia, muchos profesores expresan su frustración por la falta de importancia que se da a la promoción comercial de los medicamentos en los programas de educación de los profesionales sanitarios, y desean que aumente la importancia de este tema en la docencia [15].

Hay que destacar las limitaciones que tiene nuestro estudio. En primer lugar, los resultados de esta encuesta no son representativos de la opinión de los estudiantes en relación con la formación sobre las fuentes comerciales de medicamentos, sino que tan sólo proporcionan una experiencia local útil. En segundo lugar, no hubo un grupo de control de estudiantes que no participaran en las sesiones docentes sobre la promoción comercial de los medicamentos, y no sabemos si los estudiantes están interesados en estas actividades, independientemente del seminario, y estarían satisfechos con otras clases de actividades de enseñanza. Finalmente, no sabemos si el desarrollo de estos seminarios está asociado con una mejora de la calidad de prescripción, pero este no fue el propósito del estudio. No obstante, es necesario subrayar que pocas escuelas de medicina realizan algún tipo de evaluación de la educación sobre las fuentes comerciales de los medicamentos. La evaluación de los estudiantes proporciona una herramienta de retroalimentación útil para conocer la opinión de los estudiantes sobre los cursos y para la planificación futura.

En resumen, los estudiantes de medicina están interesados en la docencia de las fuentes de promoción comercial de los medicamentos, están satisfechos con esta actividad docente y consideran que es potencialmente útil. Estas actividades for- mativas quizá podrían influir en las actitudes de los estudiantes de medicina hacia la interacción futura con las fuentes comerciales de información de medicamentos. Mientras las fuentes principales de formación continua para los médicos estén financiadas por las empresas farmacéuticas, se necesitará una preparación universitaria sólida de los estudiantes sobre las técnicas de marketing y las limitaciones de la calidad de información que aportan las fuentes de información comercial. La investigación futura tendría que evaluar el efecto del conocimiento y las habilidades en la evaluación crítica de la información comercial de medicamentos sobre la calidad de la prescripción de éstos en la práctica clínica.

\section{Bibliografía}

1. Avorn J, Chen M, Hartley R. Scientific versus commercial sources of influence on the prescribing behaviour of physicians. Am J Med 1982; 73: 4-8.

2. Lexchin J. Interactions between physicians and the pharmaceutical industry: what dose the literature say? CMAJ 1993; 149: 1401-7.

3. Wazana A. Physician and the pharmaceutical industry: is it a gift ever just a gift? JAMA 2000; 283: 373-80.

4. Steinaman MA, Schlipak MG, McPhee SJ. Of principles and pens: attitudes and practices of medicine house staff toward pharmaceutical industry promotions. Am J Med 2001; 110: 551-7.

5. Boltri JM, Gordon ER, Vogel RL. Effect of antihypertensive samples on physician prescribing habits. Fam Med 2002; 34: 729-31.

6. Caamano F, Figueiras A, Gestal-Otero JJ. Influence of commercial information on prescription quantity in primary care. Eur J Public Health 2002; 12: 187-91.

7. Norris P, Herxheimer A, Lexchin J, Mansfield P. Drug promotion, what we know, what we have yet to learn. EDM Research Series n. ${ }^{\circ}$ 32. WHO/EDM/PAR/2004.3. URL: http://www.who.int/entity/medicines/areas/rational_use/drugPromodhai.pdf. [10.07.2007].

8. Zipkin DA, Steinman MA. Interactions between pharmaceutical representatives and doctors in training: a thematic review. J Gen Intern Med 2005; 20: 1-10.

9. Laporte JR, Porta M, Capella D, Arnau JM. Drugs in the Spanish health system. Int J Health Serv 1984; 14: 635-48.

10. Bellin M, McCarthy S, Drevlow L, Pierach C. Medical students' exposure to pharmaceutical industry market- 
ing: a survey at one U.S. medical school. Acad Med 2004; 79:1041-5.

11. Hyman PL, Hochman ME, Shaw JG, Steinman MA. Attitudes of preclinical and clinical medical students toward interactions with the pharmaceutical industry. Acad Med 2007; 82: 94-9.

12. Sierles FS, Brodkey AC, Cleary LM, McCurdy FA, Mintz $\mathrm{M}$, Frank J, et al. Medical students' exposure to and attitudes about drug company interactions: a national survey. JAMA. 2005; 294:1034-42.

13. Moghimi Y. The 'PharmFree' campaign: educating medical students about industry influence. PLoS Med 2006; 3: e30.

14. Vainiomaki M, Helve O, Vuorenkoski L. A national survey on the effect of pharmaceutical promotion on medical students. Med Teach 2004; 26: 630-4.

15. Mintzes B. Educational initiatives for medical and pharmacy students about drug promotion. EDM Research Series n. ${ }^{\circ}$ 36. WHO/PSM/PAR/2005.2. URL: http://www. who.int/entity/medicines/areas/rational_use/haipromosurvey.pdf. [10.07.2007].

16. Harvey K, Black F. Prescribing rationally, not fashionably [video]. WHO Essential Drugs Monitor 1994; 17: 23.

17. Shashindran $\mathrm{CH}$, Sethuraman KR. Drug promotion: push, promote or educate? [video]. WHO Essential Drugs Monitor 1995; 20: 24.
18. Farré M, Bosch F, Roset PN, Baños JE. Putting clinical pharmacology in context: the use of popular movies. J Clin Pharmacol 2004; 44: 30-6.

19. Wilkes MS, Hoffman JR. An innovative approach to educating medical students about pharmaceutical promotion. Acad Med 2001;76: 1271-7.

20. Wofford JL, Ohl CA. Teaching appropriate interactions with pharmaceutical company representatives: the impact of an innovative workshop on student attitudes. BMC Med Educ 2005; 5: 5.

21. Suryawati MA, Santoso B. Drug advertisements: a critical lesson for Indonesian students. WHO Essential Drugs Monitor 1997; 23: 23.

22. Vinson DC, McCandless B, Hosokawa MC. Medical students' attitudes toward pharmaceutical marketing: possibilities for change. Fam Med 1993; 25: 31-3.

23. Schneider JA, Arora V, Kasza K, Van Harrison R, Humphrey $H$. Residents' perceptions over time of pharmaceutical industry interactions and gifts and the effect of an educational intervention. Acad Med 2006; 81: 595-602.

24. Mansfield P, Lexchin J, Wen LS, Grandori L, McCoy C, Hoffman JR, et al. Educating health professionals about drug and device promotion: advocates' recommendations. PLoS Med 2006; 3: e451. 